\title{
A Multicriteria Decision-Making Approach Based on Fuzzy AHP with Intuitionistic 2-Tuple Linguistic Sets
}

\author{
Shahzad Faizi, Tabasam Rashid $\mathbb{D}$, and Sohail Zafar \\ Department of Mathematics, University of Management and Technology, Lahore 54770, Pakistan \\ Correspondence should be addressed to Tabasam Rashid; tabasam.rashid@gmail.com
}

Received 9 May 2018; Revised 26 June 2018; Accepted 9 July 2018; Published 1 August 2018

Academic Editor: Ferdinando Di Martino

Copyright (C) 2018 Shahzad Faizi et al. This is an open access article distributed under the Creative Commons Attribution License, which permits unrestricted use, distribution, and reproduction in any medium, provided the original work is properly cited.

\begin{abstract}
In the modern literature related to linguistic decision-making, the 2-tuple linguistic representation model and its useful applications in various fields have been extensively studied and used during the last decade. Recently, some useful multicriteria decision-making (MCDM) methods have been introduced based on fuzzy analytic hierarchy process (AHP) for 2-tuple linguistic representation model. By keeping in mind the importance of this linguistic model, in this paper, we introduce a fuzzy AHP methodology for intuitionistic 2-tuple linguistic sets (I2TLSs) which is a useful extension of the 2-tuple linguistic representation model. This study is comprised of four stages. In the first stage, we define some operational laws for I2TL elements (I2TLEs) and prove some related important properties. In the second stage, intuitionistic 2-tuple linguistic preference relation (I2TLPR) and multiplicative I2TLPR are defined using I2TLSs. In the 3rd stage, a transformation mechanism is introduced which can transform an I2TLPR to a corresponding intuitionistic preference relation (IPR) and vice versa. In the fourth stage, an approach is proposed for checking the consistency of an I2TLPR and presented a method to repair the inconsistent one by using the proposed transformation mechanism. Finally, a numerical example is given and comparative analysis is carried out with the TOPSIS method to verify the validity of the proposed method.
\end{abstract}

\section{Introduction}

Herrera and Martínez [1, 2] proposed the 2-tuple fuzzy linguistic representation model which can handle linguistic and numerical information in decision-making effectively without loss and distortion of information which formerly occur during the processing of linguistic information. This useful model is basically developed on the basis of symbolic translation of the linguistic variables and has been extensively used in various MCDM problems [3-6] in recent years. The basic shortcoming of this model is that it can only ensure the accuracy in dealing with uniformly distributed linguistic term sets (LTSs). To make up for the above-mentioned shortcoming, Wang and Hao [5] introduced the proportional 2-tuple fuzzy linguistic representation model, which can ensure the accuracy in dealing with the LTSs that are not uniformly distributed. The studies on MCDM problems in the context of 2-tuple fuzzy linguistic models are growing. For example, Beg and Rashid introduced two important extensions of 2-tuple linguistic representation model, namely, the hesitant 2-tuple linguistic information model [7] and the I2TL information model [8], which are very effective in dealing with fuzziness and uncertainty as compared to the ordinary 2-tuple linguistic arguments. Furthermore, Liu and Chen [9] introduced the extended T-norm and T-conorm with the I2TL information and developed a MAGDM method based on the proposed I2TL generalized aggregation operator.

AHP was originally developed by Satty in [10] which is the most powerful technique to solve complex MCDM problems and help the decision-makers (DMs) to set preferences and make the best decision. In addition, to reduce the biasness of the DMs in the decision-making process, the AHP incorporates a useful technique for checking the consistency of the DM's evaluations. Recently, extensive studies have been conducted on AHP in fuzzy context, such as, AHP based on 2-tuple linguistic representation model for supplier segmentation by aggregating quantitative and qualitative criteria [11], a hybrid approach based on 2-tuple fuzzy linguistic method and fuzzy AHP for evaluation in-flight service quality [12], 
and AHP method based on hesitant fuzzy sets for analyzing the factors affecting the performance of different branches of a cargo company [13]. To collect priorities of the DMs in AHP, different kinds of preference relations are used in the literature, but numerical preference relations [14-16] and linguistic preference relations (LPRs) $[17,18]$ are the two basic preference relations that are often used in MCDM problems. If DMs cannot guess their preferences of one alternative over the other with actual numerical values [19] and are interested in providing their preferences in linguistic values, then they prefer LPRs which are actually a kind of numerical preference relations. The LPRs have been studied as another important tool to collect preferences and have vast applications in MCDM [20-22].

To identify the inconsistency of preference relations, there is a need of a consistency check to avoid the inconsistent solutions during a decision-making process. Saaty [23] developed an idea of consistency ratio (CR) to measure the inconsistency level of numerical preference relations. $\mathrm{He}$ observed that the preference relation is of acceptable consistency if $C R<0.1$; otherwise, it is inconsistent and it is necessary to return it to the DMs again for the revision of their preferences until acceptable. Extensive studies have been done to measure the degree of inconsistency of numerical preference relations [24-26]. Similar to numerical preference relations, the consistency measure is also a difficult task while using LPRs in various MCDM problems [27]. In order to measure the consistency degree of preference relations, traditional definitions, such as the additive transitivity, the max-min transitivity, and the three-way transitivity, are used. But these definitions are incapable of measuring the consistency degree of LPRs. To make up for the above-mentioned shortcoming, Dong et al. introduced a more flexible method to measure the consistency degree of LPRs in [27]. $\mathrm{Xu}$ and Liao [28] proposed a method to check the consistency of an IPR and introduced an interesting procedure to repair the inconsistent IPR without the participation of the DM. $\mathrm{Zhu}$ and $\mathrm{Xu}$ [29] developed some consistency measures for hesitant fuzzy LPRs and further constructed two optimization methods to improve the consistency of an inconsistent hesitant fuzzy LPR. Zhang and Wu [30] discussed the multiplicative consistency of hesitant fuzzy LPRs and developed a consistency-improving process to adjust hesitant fuzzy LPR with unacceptably multiplicative consistency into an acceptably multiplicative one. Furthermore, Gong et al. [31] introduced the additive consistent conditions of the IPR according to that of intuitionistic fuzzy number preference relation. Wang [32] proved the additive consistency defined in an indirect manner in [31] and proved that the consistency transformation equations' matrix may not always be an IPR.

AHP is a widely used method for solving multicriteria problems in practical situations. The combination of AHP with fuzzy set and 2-tuple representation model can deal with human judgments under fuzzy environment and has no information loss. One of the main strengths of AHP is its ability to deal with subjective opinions of experts and derive a quantitative priority vector that describes the relative importance of each alternative, which makes AHP appealing to a wide variety of MCDM problems [33]. Some authors contend that the applicability of AHP can be attributed to its simplicity, ease of use, and flexibility as well as the possibility of integrating AHP with other techniques such as fuzzy logic and linear programming [34]. Furthermore, the role of AHP is to determine the weights of the criteria in both dimensions. This led to a consistent priority ranking with experts having to make only $\left(n^{2}-n\right) / 2$ pairwise comparisons in a decision problem containing $n$ number of alternatives. The I2TL information model is a more powerful tool in dealing with vagueness and uncertainty that can assign to each element a membership degree as well as a nonmembership degree in the form of 2-tuple linguistic information. Therefore, the aim of this study is to apply AHP method to solve MCDM problems, where the I2TL information should be collected by a tool. First, this paper has developed some operational laws for I2TLEs and proved some of the important properties related to these operational laws. The concepts of I2TLPRs and multiplicative I2TLPR are then developed to collect the preferences of the DMs as an extension of LPRs along with a transformation function that can transform an I2TLPR to a corresponding intuitionistic preference relation (IPR). Finally, an approach is proposed for checking the consistency of an I2TLPR and presented a method to repair the inconsistent one by using the proposed transformation mechanism.

The rest of the paper is organized in the following way. The preliminary concepts related to the study are briefly reviewed in Section 2. Some operational laws for I2TLEs are defined in Section 3 and their important properties are discussed with proofs. In the same section, distance measure between two I2TLEs and comparison method of I2TLEs, I2TLPR, and multiplicative I2TLPR are proposed along with a procedure to get consistent I2TLPR from the inconsistent one. In Section 4, a numerical example is given and comparative analysis is conducted with the TOPSIS method to verify the effectiveness of the proposed method. Finally, the conclusion is presented in the last section.

\section{Preliminaries}

In this section, we mainly recall some elementary concepts of LTSs and 2-tuple linguistic representation model as well as the I2TL representation model.

\subsection{Intuitionistic Fuzzy Set and Intuitionistic Preference Relation}

Definition 1 (see $[35,36])$. An IFS $\widetilde{A}$ in $X$ is given by $\widetilde{A}=$ $\left\{\left(x, \mu_{\widetilde{A}}(x), \nu_{\widetilde{A}}(x)\right) \mid x \in X\right\}$, where $\mu_{\widetilde{A}}: X \longrightarrow[0,1]$ and $\nu_{\widetilde{A}}: X \longrightarrow[0,1]$ with the condition that $0 \leq \mu_{\widetilde{A}}+\nu_{\widetilde{A}} \leq 1$ for every $x \in X$. The numbers $\mu_{\widetilde{A}}(x), \nu_{\widetilde{A}}(x) \in[0,1]$ denote, respectively, the degree of membership and nonmembership of the element $x \in X$ to the set $\widetilde{A}$. For convenience, $A=$ $\left(\mu_{A}, v_{A}\right)$ is called intuitionistic fuzzy element (IFE) and $\Omega$ the set of all IFEs. For each IFS $A$ in $X$, we will call $\pi_{A}(x)=$ $1-\mu_{A}(x)-v_{A}(x)$ the degree of indeterminacy of $x$ in $A$.

Definition 2 (see [37]). An IPR $R$ on $X=\left\{x_{1}, x_{2}, \ldots, x_{n}\right\}$ is defined by a matrix $R=\left(r_{i k}\right)_{n \times n}$, where $r_{i k}=\left(\mu\left(x_{i}, x_{k}\right)\right.$, $\left.v\left(x_{i}, x_{k}\right)\right)$ for all $1 \leq i, k \leq n$. For convenience, let $r_{i k}$ be shortly 
written as $\left(\mu_{i k}, v_{i k}\right)$, where $\mu_{i k}$ indicates the degree to which $x_{i}$ is preferred to $x_{k}$, and $v_{i k}$ indicates the degree to which $x_{i}$ is not preferred to $x_{k}$. Furthermore, $\pi\left(x_{i}, x_{k}\right)=1-\mu\left(x_{i}, x_{k}\right)-$ $v\left(x_{i}, x_{k}\right)$ denotes the indeterminacy degree or a hesitancy degree of the IPR $R$ satisfying the conditions $\mu_{i k}, v_{i k} \in[0,1]$; $\mu_{i i}=v_{i i}=0.5 ; \mu_{i k}+v_{i k} \leq 1 ; \mu_{i k}=v_{k i} ; \mu_{k i}=v_{i k} ; \pi_{i k}=1-$ $\mu_{i k}-v_{i k}$ for all $1 \leq i, k \leq n$.
2.2. Consistency Checking for Multiplicative IPR. A significant property of preference relations is multiplicative consistency. $\mathrm{Xu}$ et al. [26] proposed the definition of multiplicative consistent IPR as follows.

Definition 3 (see [26]). An IPR $R=\left(r_{i k}\right)_{n \times n}$ is multiplicative consistent with $r_{i k}=\left(\mu_{i k}, v_{i k}\right)(i, k=1,2, \ldots, n)$, if

$$
r_{i k}= \begin{cases}(0,0) & \text { if }\left(\mu_{i t}, v_{t k}\right),\left(v_{i t}, v_{t k}\right) \in\{(0,1) \\ \left(\frac{\mu_{i t} \mu_{t k}}{\mu_{i t} \mu_{t k}+\left(1-\mu_{i t}\right)\left(1-\mu_{t k}\right)}, \frac{v_{i t} v_{t k}}{v_{i t} v_{t k}+\left(1-v_{i t}\right)\left(1-v_{t k}\right)}\right) & \text { otherwise, for all } i \leq t \leq k\end{cases}
$$

For IPRs with unacceptable consistency, Xu and Liao [28] proposed a method to measure the consistency of an IPR and then introduced a method to repair the inconsistent IPR. First, they developed an algorithm to build a perfect multiplicative consistent IPR $\bar{R}=\left(\bar{r}_{i k}\right)_{n \times n}$, where $\bar{r}_{i k}=$ $\left(\bar{\mu}_{i k}, \bar{v}_{i k}\right)$ and

$$
\begin{aligned}
& \bar{\mu}_{i k} \\
& =\frac{\left(\prod_{t=i+1}^{k-1} \mu_{i t} \mu_{t k}\right)^{1 /(k-i-1)}}{\left(\prod_{t=i+1}^{k-1} \mu_{i t} \mu_{t k}\right)^{1 /(k-i-1)}+\left(\prod_{t=i+1}^{k-1}\left(1-\mu_{i t}\right)\left(1-\mu_{t k}\right)\right)^{1 /(k-i-1)}} \quad \text { for } k>i+1
\end{aligned}
$$

$\bar{v}_{i k}$

$$
\begin{aligned}
& =\frac{\left(\prod_{t=i+1}^{k-1} v_{i t} v_{t k}\right)^{1 /(k-i-1)}}{\left(\prod_{t=i+1}^{k-1} v_{i t} v_{t k}\right)^{1 /(k-i-1)}+\left(\prod_{t=i+1}^{k-1}\left(1-v_{i t}\right)\left(1-v_{t k}\right)\right)^{1 /(k-i-1)}} \\
& \text { for } k>i+1 \text {. }
\end{aligned}
$$

Definition 4 (see [28]). An IPR $R$ is called an acceptable multiplicative consistent, if the distance measure between $R$ and $\bar{R}$ denoted as $d(R, \bar{R})$ is less than $\tau$, where $\tau=0.1$ is the consistency threshold. The distance measure $d(R, \bar{R})$ can be determined as follows:

$$
\begin{aligned}
& d(R, \bar{R})=\frac{1}{2(n-1)(n-2)} \\
& \quad \cdot \sum_{i=1}^{n} \sum_{k=1}^{n}\left(\left|\bar{\mu}_{i k}-\mu_{i k}\right|+\left|\bar{\nu}_{i k}-\nu_{i k}\right|+\left|\bar{\pi}_{i k}-\pi_{i k}\right|\right)
\end{aligned}
$$

$\mathrm{Xu}$ and Liao [28] thought that the transformed IPR $\bar{R}$ cannot represent the initial preferences of the DM for a large value of $d(R, \bar{R})$. Therefore, they fused the IPRs $R$ and $\bar{R}$ into a new IPR $\widetilde{R}=\left(\widetilde{r}_{i k}\right)_{n \times n}$, where

$$
\begin{aligned}
& \tilde{\mu}_{i k}=\frac{\left(\mu_{i k}\right)^{\sigma-1}\left(\bar{\mu}_{i k}\right)^{\sigma}}{\left(1-\mu_{i k}\right)^{\sigma-1}\left(1-\bar{\mu}_{i k}\right)^{\sigma}+\left(\mu_{i k}\right)^{\sigma-1}\left(\bar{\mu}_{i k}\right)^{\sigma}} \\
& \tilde{v}_{i k}=\frac{\left(\nu_{i k}\right)^{\sigma-1}\left(\bar{v}_{i k}\right)^{\sigma}}{\left(1-\nu_{i k}\right)^{\sigma-1}\left(1-\bar{v}_{i k}\right)^{\sigma}+\left(\nu_{i k}\right)^{\sigma-1}\left(\bar{\nu}_{i k}\right)^{\sigma}}
\end{aligned}
$$

where $\sigma$ is called the controlling parameter of the IPR $\widetilde{R}$ that is set by the DM only. If $\sigma$ is small, then $\widetilde{R}$ is closer to $R$. For $\sigma=0, \widetilde{R}=R$, and for $\sigma=1, \widetilde{R}=\bar{R}$.

\subsection{Basic Concepts of Linguistic Term Set and 2-Tuple Linguistic Information}

Definition 5 (see $[38,39]$ ). Let $S=\left\{s_{0}, s_{1}, \ldots, s_{g}\right\}$ be a finite LTS with odd cardinality, where each $s_{i}(0 \leq i \leq$ g) represents a possible value for a linguistic variable. The following characteristics for $S$ can be defined as follows:

(1) Negation operator: $\operatorname{neg}\left(s_{i}\right)=s_{j}$, such that $i+j=g$;

(2) Ordered set: $s_{i} \leq s_{j} \Longleftrightarrow i \leq j$. Therefore, there exist two operators given as follows:

(a) maximization operator: $\max \left(s_{i}, s_{j}\right)=s_{i}$, if $s_{j} \leq$ $s_{i}$

(b) minimization operator: $\min \left(s_{i}, s_{j}\right)=s_{i}$, if $s_{i} \leq$ $s_{j}$.

$\mathrm{Xu}[40,41]$ introduced the concept of continuous LTS $\bar{S}$ as an extension of discrete term set $S$ where $\bar{S}=\left\{s_{k} \mid s_{0} \leq s_{k} \leq s_{g}\right\}$. The linguistic term $s_{k}$ is called the original linguistic term if $s_{k} \in S$, and is only used by the DMs to evaluate the alternatives during a decision process. If the linguistic term $s_{k} \notin S$, then $s_{k}$ is said to be the virtual linguistic term of $S$ and it appears only during the computations.

Herrera and Martínez [2] proposed the 2-tuple linguistic representation model which expresses the linguistic information by a 2-tuple $\left(s_{i}, \alpha\right)$, where $s_{i} \in S$ and $\alpha \in[-0.5,0.5)$. The basic purpose of this model is to define a transformation mechanism between linguistic 2-tuples and the numerical values.

Definition 6 (see [2]). Let $S=\left\{s_{0}, s_{1}, \ldots, s_{g}\right\}$ be a LTS and $\beta \in[0, g]$ a value representing the result of a symbolic aggregation operation. Then, a function $\triangle:[0, g] \longrightarrow S \times$ $[-0.5,0.5)$ which provides a linguistic 2 -tuple representing the equivalent information to $\beta$ is defined as follows:

$$
\triangle(\beta)=\left(s_{i}, \alpha\right), \quad \text { with } \begin{cases}s_{i}, & i=\operatorname{round}(\beta), \\ \alpha=\beta-i, & \alpha \in[-0.5,0.5) .\end{cases}
$$


Clearly, $\triangle$ is one to one function. The $\triangle$ has an inverse function $\nabla$ with $\nabla\left(\left(s_{i}, \alpha\right)\right)=i+\alpha$.

2.4. Intuitionistic 2-Tuple Linguistic Information Model. Beg and Rashid [8] proposed the idea of I2TL information model and some operators based on choquet integral to aggregate the I2TL information. They defined I2TL representation model as follows.

Definition 7 (see [8]). For a crisp set $X$ and LTS $S=$ $\left\{s_{0}, s_{1}, \ldots, s_{g}\right\}$, the set $A=\left\{\left(x, h(x), h^{\prime}(x)\right) \mid x \in X\right\}$ in $X$ where $h, h^{\prime}: X \longrightarrow S$ is called an intuitionistic LTS if $h(x)=s_{i}$ and $h^{\prime}(x)=s_{j}$ with the condition that $0 \leq i+j \leq g$, for all $x \in X$. The linguistic values $h(x)$ and $h^{\prime}(x)$ represent, respectively, the membership and nonmembership degrees of the element $x$ in the set $A$.

Definition 8 (see [8]). Let $A=\left\{\left(x, h(x), h^{\prime}(x)\right) \mid x \in X\right\}$ be intuitionistic LTS in $X$ and $\left(s_{i}, s_{j}\right) \in A$; an I2TL model can be defined as $\left(\left(s_{i}, \alpha\right),\left(s_{j}, \eta\right)\right)$, where $s_{i}, s_{j} \in S$, and $\alpha, \eta$ are numeric values in $[-0.5,0.5]$ denoting the symbolic translation of $s_{i}$ and $s_{j}$, respectively. For our convenience, $\left(\left(s_{i}, \alpha\right),\left(s_{j}, \eta\right)\right)$ is called an I2TLE and $I(S)$ is the set of all I2TLEs.

In order to avoid any loss of information, Beg and Rashid [8] further presented a computational technique to deal with this model as follows.

Definition 9 (see [8]). Let $\left(\left(s_{i}, \alpha\right),\left(s_{j}, \eta\right)\right)$ be an I2TLE for a LTS $S$. The function $\nabla:(S \times[-0.5,0.5)) \times(S \times[-0.5,0.5)) \longrightarrow$ $[-0.5, g+0.5) \times[-0.5, g+0.5)$ from $\left(\left(s_{i}, \alpha\right),\left(s_{j}, \eta\right)\right)$ to an order pair of numerical values $(\beta, \zeta) \in[-0.5, g+0.5) \times[-0.5, g+$ $0.5) \subset \mathbb{R} \times \mathbb{R}$ is defined as $\nabla\left(\left(s_{i}, \alpha\right),\left(s_{j}, \eta\right)\right)=(i+\alpha, j+\eta)=$ $(\beta, \zeta)$.

It is clear that $\beta, \zeta \in[-0.5, g+0.5)$ with the condition $0 \leq \beta+\zeta<g+1$ provided $i$ and $j$ are not simultaneously zero.

The function $\triangle:[-0.5, g+0.5) \times[-0.5, g+0.5) \longrightarrow$ $(S \times[-0.5,0.5)) \times(S \times[-0.5,0.5))$ is used to obtain the I2TL information equivalent to the pair $(\beta, \zeta)$. This function $\triangle$ can be defined as $\triangle(\beta, \zeta)=\left(\left(s_{i}, \alpha\right),\left(s_{j}, \eta\right)\right)$, where $i=\operatorname{round}(\beta)$, $j=\operatorname{round}(\eta), \alpha=\beta-i$, and $\eta=\zeta-j$. The linguistic terms $s_{i}$ and $s_{j}$ have the closest index label to $\beta$ and $\zeta$, respectively. Similarly, the values $\alpha, \eta$ represent the symbolic translations of $s_{i}$ and $s_{j}$, respectively.

\section{Operational Laws of I2TLEs and Consistency Measure}

In this section, we define some logical operational laws of I2TLEs and present some properties with proofs. The proposed operational laws for I2TLEs encompass previous operational laws for LTSs and exhibit flexibility. We also define I2TLPR and multiplicative I2TLPR and study a useful method to get a consistent I2TLPR from an inconsistent one. Furthermore, distance measure between two I2TLEs, comparison method of I2TLEs, and a methodology of I2TL AHP method are proposed in the same section to find an optimal alternative in a MCDM problem.

3.1. Some Operational Laws of I2TLEs. Gou and $\mathrm{Xu}$ [42] defined some logical operational laws for linguistic variables of a LTS on the basis of two equivalent transformation functions which can avoid the aggregated linguistic values exceeding the bounds of LTSs. They further discussed various related important properties for these operational laws. These operational laws are actually based on a transformation function $f: S \longrightarrow[0,1]$ and inverse transformation function $f^{-1}:[0,1] \longrightarrow S$ which are defined as follows:

$$
\begin{aligned}
f\left(s_{i}\right) & =\frac{i}{g}=\gamma \in[0,1], \\
f^{-1}(\gamma) & =s_{g \gamma}
\end{aligned}
$$

for all $s_{i} \in S$

Based on these transformation functions, Gou and Xu [42] introduced the following novel operational laws for linguistic values of a LTS as follows:
(1) $s_{i} \oplus s_{j}=f^{-1}\left(f\left(s_{i}\right)+f\left(s_{j}\right)-f\left(s_{i}\right) f\left(s_{j}\right)\right)$
(2) $s_{i} \otimes s_{j}=f^{-1}\left(f\left(s_{i}\right) f\left(s_{j}\right)\right)$
(3) $\lambda s_{i}=f^{-1}\left(1-\left(1-f\left(s_{i}\right)\right)^{\lambda}\right)$
(4) $s_{i}=f^{-1}\left(\left(f\left(s_{i}\right)\right)^{\lambda}\right)$

Gou and $\mathrm{Xu}$ [42] also investigated the following important properties for these novel operational laws:

(1) $s_{i} \oplus s_{j}=s_{j} \oplus s_{i}$

(2) $s_{i} \otimes s_{j}=s_{j} \otimes s_{i}$

(3) $\lambda\left(s_{i} \oplus s_{j}\right)=\lambda s_{i} \oplus \lambda s_{j}$

(4) $\left(s_{i} \otimes s_{j}\right)^{\lambda}=\left(s_{i}\right)^{\lambda} \otimes\left(s_{j}\right)^{\lambda}$

(5) $\lambda_{1} s_{i} \oplus \lambda_{2} s_{i}=\left(\lambda_{1} \oplus \lambda_{2}\right) s_{i}$

(6) $\left(s_{i}\right)^{\lambda_{1}} \otimes\left(s_{i}\right)^{\lambda_{2}}=\left(s_{i}\right)^{\lambda_{1}+\lambda_{2}}$

Motivated by the above operational laws of LTSs, we can also extend these operation laws for I2TLEs as follows.

Definition 10. Let $S=\left\{s_{0}, s_{1}, \ldots, s_{g}\right\}$ be a LTS and $I=$ $\left(\left(s_{i}, \alpha\right),\left(s_{j}, \beta\right)\right), I_{1}=\left(\left(s_{i_{1}}, \alpha_{1}\right),\left(s_{j_{1}}, \beta_{1}\right)\right)$, and $I_{2}=\left(\left(s_{i_{2}}, \alpha_{2}\right)\right.$, $\left.\left(s_{j_{2}}, \beta_{2}\right)\right)$ be three I2TLEs in $I(S)$ and $\lambda \in[0,1]$. We define

(1) $I_{1} \oplus I_{2}=\left(\left(s_{i_{1}} \oplus s_{i_{2}},\left(\alpha_{1}+\alpha_{2}\right) / 2\right),\left(s_{j_{1}} \otimes s_{j_{2}},\left(\beta_{1}+\beta_{2}\right) / 2\right)\right)$

(2) $I_{1} \otimes I_{2}=\left(\left(s_{i_{1}} \otimes s_{i_{2}},\left(\alpha_{1}+\alpha_{2}\right) / 2\right),\left(s_{j_{1}} \oplus s_{j_{2}},\left(\beta_{1}+\beta_{2}\right) / 2\right)\right)$

(3) $\lambda I=\left(\left(\lambda s_{i}, \alpha\right),\left(s_{j}^{\lambda}, \beta\right)\right)$

(4) $I^{\lambda}=\left(\left(s_{i}^{\lambda}, \alpha\right),\left(\lambda s_{j}, \alpha\right)\right)$

Theorem 11. Let $S=\left\{s_{0}, s_{1}, \ldots, s_{g}\right\}$ be a LTS and $I=$ $\left(\left(s_{i}, \alpha\right),\left(s_{j}, \beta\right)\right), I_{1}=\left(\left(s_{i_{1}}, \alpha_{1}\right),\left(s_{j_{1}}, \beta_{1}\right)\right)$, and $I_{2}=\left(\left(s_{i_{2}}, \alpha_{2}\right)\right.$, $\left.\left(s_{j_{2}}, \beta_{2}\right)\right)$ be three I2TLEs in $I(S)$ and $\lambda, \lambda_{1}, \lambda_{2} \in[0,1]$. Then 

(1) $I_{1} \oplus I_{2}=I_{2} \oplus I_{1}$
(2) $I_{1} \otimes I_{2}=I_{2} \otimes I_{1}$
(3) $\lambda\left(I_{1} \oplus I_{2}\right)=\lambda I_{1} \oplus \lambda I_{2}$
(4) $\left(I_{1} \otimes I_{2}\right)^{\lambda}=I_{1}^{\lambda} \otimes I_{2}^{\lambda}$
(5) $\lambda_{1} I \oplus \lambda_{2} I=\left(\lambda_{1}+\lambda_{2}\right) I$
(6) $I^{\lambda_{1}} \otimes I^{\lambda_{2}}=I^{\lambda_{1}+\lambda_{2}}$

Proof. (1) and (2) are simple, so the proofs of them are omitted here.

$$
\begin{aligned}
& \text { (3) } \lambda\left(I_{1} \oplus I_{2}\right)=\lambda\left(\left(s_{i_{1}} \oplus s_{i_{2}}, \frac{\alpha_{1}+\alpha_{2}}{2}\right)\right. \text {, } \\
& \left.\left(s_{j_{1}} \otimes s_{j_{2}}, \frac{\beta_{1}+\beta_{2}}{2}\right)\right)=\left(\left(\lambda\left(s_{i_{1}} \oplus s_{i_{2}}\right), \frac{\alpha_{1}+\alpha_{2}}{2}\right),\right. \\
& \left.\left(\left(s_{j_{1}} \otimes s_{j_{2}}\right)^{\lambda}, \frac{\beta_{1}+\beta_{2}}{2}\right)\right)=\left(\left(\lambda s_{i_{1}} \oplus \lambda s_{i_{2}}, \frac{\alpha_{1}+\alpha_{2}}{2}\right),\right. \\
& \left.\left(s_{j_{1}}^{\lambda} \otimes s_{j_{2}}^{\lambda}, \frac{\beta_{1}+\beta_{2}}{2}\right)\right)=\left(\left(\lambda s_{i_{1}}, \alpha_{1}\right),\left(s_{j_{1}}^{\lambda}, \beta_{1}\right)\right) \\
& \oplus\left(\left(\lambda s_{i_{2}}, \alpha_{2}\right),\left(s_{j_{2}}^{\lambda}, \beta_{2}\right)\right)=\lambda\left(\left(s_{i_{1}}, \alpha_{1}\right),\left(s_{j_{1}}, \beta_{1}\right)\right) \\
& \oplus \lambda\left(\left(s_{i_{2}}, \alpha_{2}\right),\left(s_{j_{2}}, \beta_{2}\right)\right)=\lambda I_{1} \oplus \lambda I_{2} \\
& \text { (4) }\left(I_{1} \otimes I_{2}\right)^{\lambda}=\left(\left(s_{i_{1}} \otimes s_{i_{2}}, \frac{\alpha_{1}+\alpha_{2}}{2}\right)\right. \text {, } \\
& \left.\left(s_{j_{1}} \oplus s_{j_{2}}, \frac{\beta_{1}+\beta_{2}}{2}\right)\right)^{\lambda}=\left(\left(\left(s_{i_{1}} \otimes s_{i_{2}}\right)^{\lambda}, \frac{\alpha_{1}+\alpha_{2}}{2}\right),\right. \\
& \left.\left(\lambda\left(s_{j_{1}} \oplus s_{j_{2}}\right), \frac{\beta_{1}+\beta_{2}}{2}\right)\right)=\left(\left(s_{i_{1}}^{\lambda} \otimes s_{i_{2}}^{\lambda}, \frac{\alpha_{1}+\alpha_{2}}{2}\right),\right. \\
& \left.\left(\lambda s_{j_{1}} \oplus \lambda s_{j_{2}}, \frac{\beta_{1}+\beta_{2}}{2}\right)\right)=\left(\left(s_{i_{1}}^{\lambda}, \alpha_{1}\right),\left(\lambda s_{j_{1}}, \beta_{1}\right)\right) \\
& \otimes\left(\left(s_{i_{2}}^{\lambda}, \alpha_{2}\right),\left(\lambda s_{j_{2}}, \beta_{2}\right)\right)=\left(\left(s_{i_{1}}, \alpha_{1}\right),\left(s_{j_{1}}, \beta_{1}\right)\right)^{\lambda} \\
& \otimes\left(\left(s_{i_{2}}, \alpha_{2}\right),\left(s_{j_{2}}, \beta_{2}\right)\right)^{\lambda}=I_{1}^{\lambda} \otimes I_{2}^{\lambda} \\
& \text { (5) } \lambda_{1} I \oplus \lambda_{2} I=\lambda_{1}\left(\left(s_{i}, \alpha\right),\left(s_{j}, \beta\right)\right) \oplus \lambda_{2}\left(\left(s_{i}, \alpha\right)\right. \text {, } \\
& \left.\left(s_{j}, \beta\right)\right)=\left(\left(\lambda_{1} s_{i}, \alpha\right),\left(s_{j}^{\lambda_{1}}, \beta\right)\right) \oplus\left(\left(\lambda_{2} s_{i}, \alpha\right),\left(s_{j}^{\lambda_{2}}, \beta\right)\right) \\
& =\left(\left(\lambda_{1} s_{i} \oplus \lambda_{2} s_{i}, \frac{\alpha+\alpha}{2}\right),\left(s_{j}^{\lambda_{1}} \otimes s_{j}^{\lambda_{2}}, \frac{\beta+\beta}{2}\right)\right) \\
& =\left(\left(\left(\lambda_{1}+\lambda_{2}\right) s_{i}, \alpha\right),\left(s_{j}^{\lambda_{1}+\lambda_{2}}, \beta\right)\right)=\left(\lambda_{1}+\lambda_{2}\right) \\
& \cdot\left(\left(s_{i}, \alpha\right),\left(s_{j}, \beta\right)\right)=\left(\lambda_{1}+\lambda_{2}\right) I \\
& \text { (6) } I^{\lambda_{1}} \otimes I^{\lambda_{2}}=\left(\left(s_{i}, \alpha\right),\left(s_{j}, \beta\right)\right)^{\lambda_{1}} \otimes\left(\left(s_{i}, \alpha\right),\left(s_{j}, \beta\right)\right)^{\lambda_{2}} \\
& =\left(\left(s_{i}^{\lambda_{1}}, \alpha\right),\left(\lambda_{1} s_{j}, \beta\right)\right) \otimes\left(\left(s_{i}^{\lambda_{2}}, \alpha\right),\left(\lambda_{2} s_{j}, \beta\right)\right) \\
& =\left(\left(s_{i}^{\lambda_{1}} \otimes s_{i}^{\lambda_{2}}, \frac{\alpha+\alpha}{2}\right),\left(\lambda_{1} s_{j} \oplus \lambda_{2} s_{j}, \frac{\beta+\beta}{2}\right)\right)
\end{aligned}
$$

$$
\begin{aligned}
& =\left(\left(s_{i}^{\lambda_{1}+\lambda_{2}}, \alpha\right),\left(\left(\lambda_{1}+\lambda_{2}\right) s_{j}, \beta\right)\right)=\left(\left(s_{i}, \alpha\right),\right. \\
& \left.\left(s_{j}, \beta\right)\right)^{\lambda_{1}+\lambda_{2}}=I^{\lambda_{1}+\lambda_{2}}
\end{aligned}
$$

In the following, we put forward the axiom of distance measure for I2TLEs, shown as follows:

Definition 12. Let $S=\left\{s_{0}, s_{1}, \ldots, s_{g}\right\}$ be a LTS and $I_{1}=$ $\left(\left(s_{i_{1}}, \alpha_{1}\right),\left(s_{j_{1}}, \beta_{1}\right)\right)$ and $I_{2}=\left(\left(s_{i_{2}}, \alpha_{2}\right),\left(s_{j_{2}}, \beta_{2}\right)\right)$ be two I2TLEs in $I(S)$. The Euclidean distance $d_{e d}$ between $I_{1}$ and $I_{2}$ can be defined as follows:

$$
\begin{aligned}
& d_{g d}\left(I_{1}, I_{2}\right)=\frac{1}{\sqrt{2} g} \\
& \quad \cdot \sqrt{\left(\left(i_{1}+\alpha_{1}\right)-\left(i_{2}+\alpha_{2}\right)\right)^{2}+\left(\left(j_{1}+\beta_{1}\right)-\left(j_{2}+\beta_{2}\right)\right)^{2}}
\end{aligned}
$$

Definition 13. Let $I_{1}, I_{2} \in I(S)$; then the Euclidean distance $d_{e d}$ between $I_{1}$ and $I_{2}$ satisfies the following:

(1) $0 \leq d_{e d}\left(I_{1}, I_{2}\right) \leq 1$;

(2) $d_{e d}\left(I_{1}, I_{2}\right)=0$ if and only if $I_{1}=I_{2}$;

(3) $d_{e d}\left(I_{1}, I_{2}\right)=d_{e d}\left(I_{2}, I_{1}\right)$.

Liu and Chen [9] proposed score and accuracy functions for the comparison of two I2TLEs. We now introduce a new comparison method for I2TLEs, which can be seen as follows.

Definition 14. Let $I=\left(\left(s_{i}, \alpha\right),\left(s_{j}, \eta\right)\right)$ be an I2TLE in $I(S)$ where $\alpha, \eta \notin[-0.5,0)$ for $i=j=0$. The score function $S(I)$, the accuracy function $A c(I)$, and hesitancy degree value $h(I)$ of $I$ can be defined as follows:

$$
\begin{aligned}
S(I) & =\frac{1}{2 g}((i+\alpha)-(j+\eta)+g) \\
A c(I) & =\frac{1}{2 g}((i+\alpha)+(j+\eta)+g)
\end{aligned}
$$

It can be observed that $S(I), A c(I) \in[0,1]$. The comparison method of I2TLEs based on score and accuracy functions can be established as follows.

Definition 15. For any two I2FLEs $I_{1}, I_{2} \in I(S)$,

(1) if $S\left(I_{1}\right)>S\left(I_{2}\right)$, then $I_{1}>I_{2}$;

(2) if $S\left(I_{1}\right)=S\left(I_{2}\right)$, and

(a) $A c\left(I_{1}\right)>A c\left(I_{2}\right)$, then $I_{1}>I_{2}$;

(b) $A c\left(I_{1}\right)=A c\left(I_{2}\right)$, then $I_{1}=I_{2}$

Example 16. Let $S=\left\{s_{0}, s_{1}, \ldots, s_{6}\right\}$ be a LTS and $I_{1}=$ $\left(\left(s_{2},-0.2\right),\left(s_{3}, 0\right)\right)$ and $I_{2}=\left(\left(s_{2}, 0.2\right),\left(s_{3},-0.5\right)\right)$ be two I2TLEs in $I(S)$. Then using (11), $S\left(I_{1}\right)=0.4000, S\left(I_{2}\right)=$ 0.4750. This shows that $I_{2}>I_{1}$. Similarly, for $I_{3}=$ $\left(\left(s_{4}, 0.1\right),\left(s_{1},-0.3\right)\right), I_{4}=\left(\left(s_{4}, 0.2\right),\left(s_{1},-0.2\right)\right)$ in $I(S), S\left(I_{3}\right)=$ $S\left(I_{4}\right)=0.7833$ but $A c\left(I_{3}\right)=0.9$ and $A c\left(I_{4}\right)=0.9167$. This implies $I_{4}>I_{3}$. 
TABLE 1: The RI $(n)$ values for $n \leq 10$.

\begin{tabular}{lccccccccc}
\hline$n$ & 2 & 3 & 4 & 5 & 6 & 7 & 8 & 9 & 10 \\
\hline$R I(n)$ & 0 & 0.58 & 0.90 & 1.12 & 1.24 & 1.32 & 1.41 & 1.45 & 1.51 \\
\hline
\end{tabular}

3.2. Consistency Measure of I2TLPR. In preference relations, consistency is an important topic in decision-making and the lack of consistency can lead to inconsistent solutions. Some inconsistencies may typically arise while finding consistent solution to MCDM problems when many pairwise comparisons are performed by the DMs during assessment processes. Saaty [23] proposed a consistency index and a consistency ratio denoted as " $C I$ " and " $C R$ ", respectively, in the conventional AHP method to compute the degree or level of consistency for a multiplicative preference relation by using the following formulae:

$$
\begin{aligned}
C I & =\frac{\lambda_{\max }-n}{n-1} \\
C R & =\frac{C I}{R I(n)}
\end{aligned}
$$

where $\lambda_{\max }$ and $n$ are, respectively, the largest eigenvalue and the dimension of the multiplicative preference relation. The term $R I(n)$ is denoted as random index that completely depends on the value of $n$. The values of $R I(n)$ for $n \leq 10$ are shown in Table 1 . The value of $C I$ is always equal to zero for a perfectly consistent DM, but small values of inconsistency may be tolerated during a decision process. However, perfect consistency rarely occurs in practice.

Saaty [23] identified that the multiplicative preference relation is of acceptable level of consistency when $C R<0.1$; otherwise, it is inconsistent and it is necessary to return it to the DMs again for the revision of their preferences until they are acceptable.

Due to the importance of consistent preference relations, we now focus on the studies of the consistency measures of I2TLPRs. First, we will define I2TLPR and the multiplicative I2TLPR and then propose an intuitionistic 2-tuple transformation function which is useful in obtaining an consistent I2TLPR.

\subsubsection{Intuitionistic 2-Tuple Linguistic Preference Relation}

Definition 17. Let $X=\left\{x_{1}, x_{2}, \ldots, x_{n}\right\}$ be a fixed given set of alternatives and $S=\left\{s_{0}, s_{1}, \ldots, s_{g}\right\}$ a LTS. Suppose the DMs provide their pairwise comparison assessments of alternatives by linguistic values based on $S$ and the numeric values representing the symbolic translation are selected from the interval $[-0.5,0.5)$, and these linguistic values along with symbolic translation are transformed into I2TLSs. The I2TLPR can be defined as follows.

An I2TLPR $B$ is denoted by a matrix $B=\left(b_{i j}\right)_{n \times n}$, where

$b_{i j}=\left(\left(x_{i}, x_{j}\right), b_{m}^{i j}\left(x_{i}, x_{j}\right), b_{n}^{i j}\left(x_{i}, x_{j}\right)\right), b_{m}^{i j}=\left(s_{p}^{i j}, \alpha_{p}^{i j}\right)$, and $b_{n}^{i j}=\left(s_{q}^{i j}, \eta_{q}^{i j}\right)$. For convenience, we let $b_{i j}=\left(\left(s_{p}^{i j}, \alpha_{p}^{i j}\right),\left(s_{q}^{i j}\right.\right.$, $\left.\left.\eta_{q}^{i j}\right)\right)=\left(b_{m}^{i j}, b_{n}^{i j}\right)$, where $0 \leq p+q \leq g$ and $\alpha_{p}^{i j}, \eta_{q}^{i j} \in[-0.5,0.5)$. $b_{m}^{i j}$ denotes the degree to which $x_{i}$ is preferred to $x_{j} ; b_{n}^{i j}$ indicates the degree to which $x_{i}$ is not preferred to $x_{j}$. For all $i, j=1,2, \ldots, n, b_{i j}(i<j)$, the I2TLPR should satisfy the following conditions: $b_{m}^{i j}, b_{n}^{i j}$ represent, respectively, the linguistic information by 2-tuple $\left(s_{p}^{i j}, \alpha_{p}^{i j}\right)$ and $\left(s_{q}^{i j}, \eta_{q}^{i j}\right), b_{m}^{i j}=$ $b_{n}^{j i}, b_{m}^{j i}=b_{n}^{i j}, b_{m}^{i i}=b_{n}^{j j}=\left(\left(s_{3}, 0.5\right),\left(s_{3}, 0.5\right)\right)$ for all $i, j=$ $1,2, \ldots, n$.

3.2.2. Intuitionistic 2-Tuple Transformation Function. In order to define a multiplicative I2TLPR, we first define a transformation function which can transform an I2TLE to an element of $\Omega$ and then the inverse transformation function as follows.

Definition 18. Let $I=\left(\left(s_{i}, \alpha\right),\left(s_{j}, \eta\right)\right)$ be an intuitionistic 2-tuple. We define a intuitionistic 2-tuple transformation function $h: I(S) \longrightarrow \Omega^{\prime} \subset \Omega$ as $h(I)=(\beta /(g+1), \zeta /(g+1))=$ $(a, b)$, where $\beta=i+\alpha$ and $\zeta=j+\eta$ and $\alpha, \eta \notin[-0.5,0)$ for $i=j=0$. Clearly $0 \leq a+b=(\beta+\zeta) /(g+1)<(g+1) /(g+1)=1$ because $0 \leq \beta+\zeta<g+1$. It should be noted here that, as $\alpha+\eta \longrightarrow 1, a+b \longrightarrow 1$ for $i=g$ and $j=0$ or $i=0$ and $j=g$. Similarly, we can define the intuitionistic 2-tuple inverse transformation function $h^{-1}: \Omega^{\prime} \longrightarrow I(S)$ as $h^{-1}(a, b)=\left(\left(s_{i}, \alpha\right),\left(s_{j}, \eta\right)\right) \in I(S)$, where $i=\operatorname{round}((g+1) a)$, $j=\operatorname{round}((g+1) b), \alpha=(g+1) a-i$, and $\eta=(g+1) b-j$ for any $(a, b) \in \Omega^{\prime}$.

Example 19. Let $S=\left\{s_{0}, s_{1}, \ldots, s_{6}\right\}$ be a LTS. Suppose $I=\left(\left(s_{5}, 0.2\right),\left(s_{1}-0.5\right)\right)$ is an I2TLE. Then, $h(I)=$ $(0.74290 .0714) \in \Omega^{\prime}$ is the corresponding IFE. Similarly, by applying intuitionistic 2-tuple inverse transformation function, we get $h^{-1}(0.7429,0.0714)=I$.

Remark 20. The intuitionistic 2-tuple transformation mechanism can provide a relationship between intuitionistic 2tuples and IFEs. Obviously, it is convenient to obtain the transformation results according to different situations of decision-making processes.

Remark 21. The intuitionistic 2-tuple transformation mechanism provides a useful relationship between intuitionistic 2tuples and IFEs. Therefore, the values of parameters $\tau$ and $\sigma$ can be used as discussed in Section 2.2 during the process of obtaining a consistent I2TLPR. Moreover, Table 1 can also be used as it is during the computation process of consistency measure of I2TLPR.

Definition 22. An I2TLPR $B=\left(b_{i j}\right)_{n \times n}$ with $b_{i j}=\left(b_{m}^{i j}, b_{n}^{i j}\right)$, $b_{m}^{i j}=\left(s_{p}^{i j}, \alpha_{p}^{i j}\right), b_{n}^{i j}=\left(s_{q}^{i j}, \eta_{q}^{i j}\right), 0 \leq p+q \leq g$, and $\alpha_{p}^{i j}, \eta_{q}^{i j} \epsilon$ $[-0.5,0.5)$ is multiplicative consistent if its corresponding IPR $h(B)=\left(h\left(b_{i j}\right)\right)_{n \times n}$ is multiplicative consistent.

3.2.3. How to Find the Consistent Multiplicative Consistent I2TLPR. For I2TLPR $B=\left(b_{i j}\right)_{n \times n}$, our aim is now to let $B$ approach a consistent one without the interaction of DMs. 
TABLE 2: I2TLPR of criteria concerning the overall objective.

\begin{tabular}{lcccc}
\hline$B$ & $C_{1}$ & $C_{2}$ & $C_{3}$ & $C_{4}$ \\
\hline$C_{1}$ & $\left(\left(s_{3}, 0.5\right)\right.$, & $\left(\left(s_{4}, 0.3\right)\right.$, & $\left(\left(s_{3}, 0.2\right)\right.$, & $\left(\left(s_{4},-0.45\right)\right.$, \\
& $\left.\left(s_{3}, 0.5\right)\right)$ & $(s 1,0.4))$ & $\left.\left(s_{2}, 0.3\right)\right)$ & $\left.\left(s_{2},-0.3\right)\right)$ \\
$C_{2}$ & $\left(\left(s_{1}, 0.4\right)\right.$, & $\left(\left(s_{3}, 0.5\right)\right.$, & $\left(\left(s_{3}, 0.2\right)\right.$, & $\left(\left(s_{4},-0.45\right)\right.$, \\
& $\left.\left(s_{4}, 0.3\right)\right)$ & $\left.\left(s_{3}, 0.5\right)\right)$ & $\left.\left(s_{1}, 0.4\right)\right)$ & $\left.\left(s_{0}, 0.2\right)\right)$ \\
$C_{3}$ & $\left(\left(s_{2}, 0.3\right)\right.$, & $\left(\left(s_{1}, 0.4\right)\right.$, & $\left(\left(s_{3}, 0.5\right)\right.$, & $\left(\left(s_{4}, 0.2\right)\right.$, \\
& $\left.\left(s_{3}, 0.2\right)\right)$ & $\left.\left(s_{3}, 0.2\right)\right)$ & $\left.\left(s_{3}, 0.5\right)\right)$ & $\left(\left(s_{1}, 0.3\right)\right)$ \\
$C_{4}$ & $\left(\left(s_{2},-0.3\right)\right.$, & $\left(\left(s_{0}, 0.2\right)\right.$, & $\left(s_{3}, 0.3\right)$, & $\left.\left(s_{3}, 0.5\right)\right)$ \\
& $\left.\left(s_{4},-0.45\right)\right)$ & $\left.\left(s_{4},-0.45\right)\right)$ & & \\
\hline
\end{tabular}

The following algorithm is developed to obtain a consistent I2TLPR $B$ if $B$ is of unacceptable consistency.

\section{Algorithm 23.}

Step 1. Assuming an I2TLPR $B=\left(b_{i j}\right)_{n \times n}$, and the predefined consistent threshold $\tau$, obtain a corresponding IPR $R=$ $h(B)=\left(h\left(b_{i j}\right)\right)_{n \times n}$ by using the I2T transformation function (see Definition 18).

Step 2. Suppose that $y$ is the number of iterations. Let $y=1$, and construct a perfect multiplicative consistent IPR $\bar{R}$ from $R_{y}=R$ using (2)-(4).

Step 3. Construct estimated consistency of $R_{y}$ by computing $d\left(\bar{R}, R_{y}\right)$ using (4). If $d\left(\bar{R}, R_{y}\right) \leq \tau$, then output $R_{y}$; otherwise, go to the next step.

Step 4. By using (5) and (6), construct the fused IPR $\bar{R}_{y}$ by letting a suitable value of the controlling parameter $\sigma$.

Step 5. Compute $d\left(\bar{R}_{y}, R\right)$ using (4). If $d\left(\bar{R}_{y}, R\right) \leq \tau$, then output $\bar{R}_{y}$; otherwise, repeat Step 3 .

Step 6. Set $R_{y+1}=\bar{R}_{y}$. Let $y=y+1$, and, then, go to the next step.

Step 7. Construct the corresponding consistent I2TLPR $B^{c}=$ $h^{-1}\left(\bar{R}_{y}\right)$ (see Definition 18 ).

3.3. Intuitionistic 2-Tuple Linguistic AHP Method. Let $A=$ $\left\{A_{1}, A_{2}, \ldots, A_{m}\right\}$ be a discrete set of $m$ possible alternatives and a set of $n$ objective criteria $C=\left\{C_{1}, C_{2}, \ldots, C_{n}\right\}$. Now we formulate the I2TL AHP model to solve MCDM problems. The next six steps can sum up the whole procedure of applying the I2TL AHP method.

Step 1. Construct a hierarchical structure for the decision problem to be solved.

Step 2. Establish the I2TLPR $B=\left(b_{p q}\right)_{n \times n}$ through the pairwise comparison between each criterion. At the same time, further I2TLPRs $B_{j}=\left(b_{p q}^{j}\right)_{m \times m}(j=1,2, \ldots, n)$ of alternatives concerning the criteria $C_{j}(j=1,2, \ldots, n)$ are constructed via the pairwise comparison of alternatives under each criterion.
Step 3. Check the consistency degree of each I2TLPR $B$ and $B_{j}(j=1,2, \ldots, n)$ as discussed in Step 4. If all I2TLPRs are already of acceptable consistency, ignore Step 4.

Step 4. Repair the inconsistent I2TLPRs $B$ and $B_{j}(j=$ $1,2, \ldots, n$ ) by using Algorithm 23 (or return them to the DM for reconsideration until they are acceptable).

Step 5. Calculate the aggregated criteria weights vector $W_{C}=$ $\left(w_{1}^{c}, w_{2}^{c}, \ldots, w_{n}^{c}\right)$ of I2TLPR using Definition 10, Part (1). Similarly, also calculate the aggregated weight vector $W_{A}=$ $\left(w_{1}^{A}, w_{2}^{A}, \ldots, w_{m}^{A}\right)$ of the alternatives by using the same definition.

Step 6. Rank the overall weights $w_{i}^{A}(i=1,2, \ldots, m)$ of each alternative using Definition 14 , and then choose the best alternative.

\section{Numerical Example}

Based on the availability of information and the scope to get direct, prompt, and appealing information, each student is more willing to select a university option of his/her interest that exactly answers the questions and how the accessibility of this information determine whether one will select one university option over the other. For this, portals of three different universities of Pakistan $A_{1}, A_{2}$, and $A_{3}$ are evaluated under the four criteria: $C_{1}$ : simple and professional design; $C_{2}$ : student services; $C_{3}$ : research interface; and $C_{4}$ : alumni section.

The three alternatives $A_{i}(i=1,2,3)$ are evaluated by a DM using the LTS $S=\left\{s_{0}=\right.$ Extremely poor, $s_{1}=$ Very Poor, $s_{2}=$ Poor, $s_{3}=$ Medium, $s_{4}=$ Good, $s_{5}=$ Very Good, and $s_{6}=$ Extremely Good $\}$ under the above four criteria. In the following, we use our proposed intuitionistic 2-tuple AHP method to get the best alternative as follows.

The comparison judgments of the priority of one criterion over the other determined by the DM are represented in I2TLPR $B=\left(b_{p q}\right)_{4 \times 4}$ and shown in Table 2 .

Similarly, the comparison judgments of the priority of one alternative over the remaining are represented in I2TLPRs $B_{j}=\left(b_{p q}^{j}\right)_{3 \times 3}(j=1,2, \ldots, n)$ and shown in Tables 3-6.

Now, we check the consistency level of each I2TLPR by following the idea presented by $\mathrm{Xu}$ and Liao in [28, Algorithm 1]. 
TABLE 3: I2TLPR of alternatives concerning the criterion $C_{1}$.

\begin{tabular}{lccr}
\hline$B_{1}$ & $A_{1}$ & $A_{2}$ & $A_{3}$ \\
\hline$A_{1}$ & $\left(\left(s_{3}, 0.5\right),\left(s_{3}, 0.5\right)\right)$ & $\left(\left(s_{1}, 0.4\right),\left(s_{4}, 0.2\right)\right)$ & $\left(\left(s_{3},-0.5\right),\left(s_{2},-0.2\right)\right)$ \\
$A_{2}$ & $\left(\left(s_{4}, 0.2\right),\left(s_{1}, 0.4\right)\right)$ & $\left(\left(s_{3}, 0.5\right),\left(s_{3}, 0.5\right)\right)$ & $\left(\left(s_{3}, 0.1\right),\left(s_{2},-0.2\right)\right)$ \\
$A_{3}$ & $\left(\left(s_{2},-0.2\right),\left(s_{3},-0.5\right)\right)$ & $\left(\left(s_{2},-0.2\right),\left(s_{3}, 0.1\right)\right)$ & $\left(\left(s_{3}, 0.5\right),\left(s_{3}, 0.5\right)\right)$ \\
\hline
\end{tabular}

TABLE 4: I2TLPR of alternatives concerning the criterion $C_{2}$.

\begin{tabular}{lccc}
\hline$B_{2}$ & $A_{1}$ & $A_{2}$ & $A_{3}$ \\
\hline$A_{1}$ & $\left(\left(s_{3}, 0.5\right),\left(s_{3}, 0.5\right)\right)$ & $\left(\left(s_{1}, 0.4\right),\left(s_{4}, 0.2\right)\right)$ & $\left(\left(s_{3}, 0.45\right),\left(s_{1}, 0.4\right)\right)$ \\
$A_{2}$ & $\left(\left(s_{4}, 0.2\right),\left(s_{1}, 0.4\right)\right)$ & $\left(\left(s_{3}, 0.5\right),\left(s_{3}, 0.5\right)\right)$ & $\left(\left(s_{3}, 0.15\right),\left(s_{2},-0.2\right)\right)$ \\
$A_{3}$ & $\left(\left(s_{1}, 0.4\right),\left(s_{3}, 0.45\right)\right)$ & $\left(\left(s_{2},-0.2\right),\left(s_{3}, 0.15\right)\right)$ & $\left(\left(s_{3}, 0.5\right),\left(s_{3}, 0.5\right)\right)$ \\
\hline
\end{tabular}

TABLE 5: I2TLPR of alternatives concerning the criterion $C_{3}$.

\begin{tabular}{lccc}
\hline$B_{3}$ & $A_{1}$ & $A_{2}$ & $A_{3}$ \\
\hline$A_{1}$ & $\left(\left(s_{3}, 0.5\right),\left(s_{3}, 0.5\right)\right)$ & $\left(\left(s_{1},-0.25\right),\left(s_{4},-0.15\right)\right)$ & $\left(\left(s_{4}, 0.2\right),\left(s_{1}, 0.4\right)\right)$ \\
$A_{2}$ & $\left(\left(s_{4},-0.15\right),\left(s_{1},-0.25\right)\right)$ & $\left(\left(s_{3}, 0.5\right),\left(s_{3}, 0.5\right)\right)$ & $\left(\left(s_{3},-0.45\right),\left(s_{1},-0.3\right)\right)$ \\
$A_{3}$ & $\left(\left(s_{1}, 0.1\right),\left(s_{4}, 0.2\right)\right)$ & $\left(\left(s_{1},-0.3\right),\left(s_{3},-0.45\right)\right)$ & $\left(\left(s_{3}, 0.5\right),\left(s_{3}, 0.5\right)\right)$ \\
\hline
\end{tabular}

Suppose we take the I2TLPR $B$ of criteria as an example and discuss the process of checking the consistency.

$$
\bar{B}=\left(\begin{array}{cccc}
\left(\left(s_{3}, 0.5\right),\right. & \left(\left(s_{4}, 0.3\right),\right. & \left(\left(s_{4},-0.2436\right),\right. & \left(\left(s_{4}, 0.1297\right),\right. \\
\left.\left(s_{3}, 0.5\right)\right) & \left.\left(s_{1}, 0.4\right)\right) & \left.\left(s_{1}, 0.4\right)\right) & \left.\left(s_{0}, 0.1949\right)\right) \\
\left(\left(s_{1}, 0.4\right),\right. & \left(\left(s_{3}, 0.5\right),\right. & \left(\left(s_{3}, 0.2\right),\right. & \left(\left(s_{4},-0.2958\right),\right. \\
\left.\left(s_{4}, 0.3\right)\right) & \left.\left(s_{3}, 0.5\right)\right) & \left.\left(s_{1}, 0.4\right)\right) & \left.\left(s_{1}, 0.3493\right)\right) \\
\left(\left(s_{1}, 0.4\right),\right. & \left(\left(s_{1}, 0.4\right),\right. & \left(\left(s_{3}, 0.5\right),\right. & \left(\left(s_{4}, 0.2\right),\right. \\
\left.\left(s_{4},-0.2436\right)\right) & \left.\left(s_{3}, 0.2\right)\right) & \left.\left(s_{3}, 0.5\right)\right) & \left.\left(s_{1}, 0.3\right)\right) \\
\left(\left(s_{0}, 0.1949\right),\right. & \left(\left(s_{1}, 0.3493\right),\right. & \left(\left(s_{1}, 0.3\right),\right. & \left(\left(s_{3}, 0.5\right),\right. \\
\left.\left(s_{4}, 0.1297\right)\right) & \left.\left(s_{4},-0.2958\right)\right) & \left.\left(s_{4}, 0.2\right)\right) & \left.\left(s_{3}, 0.5\right)\right)
\end{array}\right)
$$

By finding the distance between $R$ and $\bar{R}$ corresponding to the I2TLPRs $B$ and $\bar{B}$ with the help of (4), we get $d(R, \bar{R})=$ 0.1766 which is greater than 0.1 , which means the I2TLPR $\bar{B}$ is of unacceptable consistency and, therefore, it is necessary
The perfect multiplicative consistent I2TLPR $\bar{B}=\left(\bar{b}_{i j}\right)_{4 \times 4}$ of the I2TLPR $B$ of criteria can be constructed as

to repair it. To improve the consistency, (2.4) and (2.5) in [28, Algorithm 2] are used to get the fused I2TLPR $\bar{B}_{1}$ by letting $\sigma=0.8$ as follows:

$$
\bar{B}_{1}=\left(\begin{array}{cccc}
\left(\left(s_{3}, 0.5\right),\right. & \left(\left(s_{4}, 0.3\right),\right. & \left(\left(s_{4},-0.3548\right),\right. & \left(\left(s_{4}, 0.0155\right),\right. \\
\left.\left(s_{3}, 0.5\right)\right) & \left.\left(s_{1}, 0.4\right)\right) & \left.\left(s_{2},-0.4435\right)\right) & \left.\left(s_{0}, 0.3107\right)\right) \\
\left(\left(s_{1}, 0.4\right),\right. & \left(\left(s_{3}, 0.5\right),\right. & \left(\left(s_{3}, 0.2\right),\right. & \left(\left(s_{4},-0.3266\right),\right. \\
\left.\left(s_{4}, 0.3\right)\right) & \left.\left(s_{3}, 0.5\right)\right) & \left.\left(s_{1}, 0.4\right)\right) & \left.\left(s_{1},-0.0497\right)\right) \\
\left(\left(s_{2},-0.4435\right),\right. & \left(\left(s_{1}, 0.4\right),\right. & \left(\left(s_{3}, 0.5\right),\right. & \left(\left(s_{4}, 0.2\right),\right. \\
\left.\left(s_{4},-0.3548\right)\right) & \left.\left(s_{3}, 0.2\right)\right) & \left.\left(s_{3}, 0.5\right)\right) & \left.\left(s_{1}, 0.3\right)\right) \\
\left(\left(s_{0}, 0.3107\right),\right. & \left(\left(s_{1},-0.0497\right),\right. & \left(\left(s_{1}, 0.3\right),\right. & \left(\left(s_{3}, 0.5\right),\right. \\
\left.\left(s_{4}, 0.0155\right)\right) & \left.\left(s_{4},-0.3266\right)\right) & \left.\left(s_{4}, 0.2\right)\right) & \left.\left(s_{3}, 0.5\right)\right)
\end{array}\right)
$$


TABLE 6: I2TLPR of alternatives concerning the criterion $C_{4}$.

\begin{tabular}{lccc}
\hline$B_{4}$ & $A_{1}$ & $A_{2}$ & $A_{3}$ \\
\hline$A_{1}$ & $\left(\left(s_{3}, 0.5\right),\left(s_{3}, 0.5\right)\right)$ & $\left(\left(s_{1},-0.25\right),\left(s_{4},-0.15\right)\right)$ & $\left(\left(s_{4}, 0.2\right),\left(s_{1}, 0.4\right)\right)$ \\
$A_{2}$ & $\left(\left(s_{4},-0.15\right),\left(s_{1},-0.25\right)\right)$ & $\left(\left(s_{3}, 0.5\right),\left(s_{3}, 0.5\right)\right)$ & $\left(\left(s_{3},-0.45\right),\left(s_{1},-0.3\right)\right)$ \\
$A_{3}$ & $\left(\left(s_{1}, 0.1\right),\left(s_{4}, 0.2\right)\right)$ & $\left(\left(s_{1},-0.3\right),\left(s_{3},-0.45\right)\right)$ & $\left(\left(s_{3}, 0.5\right),\left(s_{3}, 0.5\right)\right)$ \\
\hline
\end{tabular}

TABLE 7: Over all aggregated weights of alternatives and criteria.

\begin{tabular}{ccccc}
\hline & $w_{j}^{c}$ & $w_{j}^{A_{1}}$ & $w_{j}^{A_{2}}$ & $w_{j}^{A_{3}}$ \\
\hline$C_{1}$ & $\left(\left(s_{5.8889}, 0.0190\right)\right.$, & $\left(\left(s_{4.75},-0.0250\right)\right.$, & $\left(\left(s_{5.5}, 0.2250\right)\right.$, & $\left(\left(s_{4.6667}, 0.15\right)\right.$, \\
& $\left.\left(s_{0}, 0.1570\right)\right)$ & $\left.\left(s_{0.6667}, 0.0750\right)\right)$ & $\left.\left(s_{0.1667}, 0.1250\right)\right)$ & $\left.\left(s_{0.75}, 0.15\right)\right)$ \\
$C_{2}$ & $\left(\left(s_{5.5833},-0.0008\right)\right.$, & $\left(\left(s_{4.75},-0.0250\right)\right.$, & $\left(\left(s_{5.5}, 0.2250\right)\right.$, & $\left(\left(s_{4.6667}, 0.15\right)\right.$, \\
& $\left.\left(s_{0.0556}, 0.1751\right)\right)$ & $\left.\left(s_{0.6667}, 0.0750\right)\right)$ & $\left.\left(s_{0.1667}, 0.1250\right)\right)$ & $\left.\left(s_{0.75}, 0.15\right)\right)$ \\
$C_{3}$ & $\left(\left(s_{5.4444}, 0.2195\right)\right.$, & $\left(\left(s_{5.1667}, 0.1625\right)\right.$, & $\left(\left(s_{5.5},-0.1375\right)\right.$, & $\left(\left(s_{3.9167}, 0.2\right)\right.$, \\
& $\left.\left(s_{0.1667}, 0.2556\right)\right)$ & $\left.\left(s_{0.3333}, 0.1375\right)\right)$ & $\left.\left(s_{0.0833},-0.0875\right)\right)$ & $\left.\left(s_{1}, 0.1875\right)\right)$ \\
$C_{4}$ & $\left(\left(s_{3.9167}, 0.3576\right)\right.$, & $\left(\left(s_{5.5833},-0.0250\right)\right.$, & $\left(\left(s_{5.5},-0.0250\right)\right.$, & $\left(\left(s_{3.9167}, 0.2999\right)\right.$, \\
& $\left.\left(s_{0.8889}, 0.2611\right)\right)$ & $\left.\left(s_{0.3333},-0.0750\right)\right)$ & $\left.\left(s_{0.0833}, 0.25\right)\right)$ & $\left.\left(s_{1.25}, 0.1875\right)\right)$ \\
\hline
\end{tabular}

Corresponding to the I2TLPRs $B$ and $\bar{B}_{1}$, the distance between $R$ and $\bar{R}_{1}$ is calculated as $d\left(R, \bar{R}_{1}\right)=0.0334$ with the help of (4) again, which is now less than 0.1. This means $\bar{B}_{1}$ is of acceptable multiplicative consistency. For the other intuitionistic preference relations $B_{j}(j=1,2,3,4)$ of alternatives concerning the criteria $C_{j}(j=1,2,3,4)$, the consistency checking can be done by following the same process. We can see that all the other I2TLPRs are consistent, and we do not need to repair them.

Now, by using Definition 10, Part (1), we can calculate the aggregated criteria weights vector $W_{C}=\left(w_{1}^{c}, w_{2}^{c}, \ldots, w_{n}^{c}\right)$ of the I2TLPR $\bar{B}_{1}$ as

$$
\begin{aligned}
& w_{1}^{c}=\left(\left(s_{5.8889}, 0.0190\right),\left(s_{0}, 0.1570\right)\right) \\
& w_{2}^{c}=\left(\left(s_{5.5833},-0.0008\right),\left(s_{0.0556}, 0.1751\right)\right) \\
& w_{3}^{c}=\left(\left(s_{5.4444}, 0.2195\right),\left(s_{0.1667}, 0.2556\right)\right), \\
& w_{4}^{c}=\left(\left(s_{3.9167}, 0.3576\right),\left(s_{0.8889}, 0.2611\right)\right)
\end{aligned}
$$

Similarly, the aggregated weight vectors of the other I2TLPRs $B_{j}(j=1,2,3,4)$ of alternatives concerning the criteria $C_{j}(j=1,2,3,4)$ are calculated by using again Definition 10, Part 1. The aggregated results are as shown in Table 7.

At the end, we aggregate all the criteria weights and weights of alternatives as computed above by using the operational laws discussed in Definition 10 concerning each alternative as follows:

$$
\begin{aligned}
w_{1}^{A} & =\bigoplus_{j=1}^{4}\left(w_{j}^{c} \otimes w_{j}^{A}\right)=\left(w_{1}^{c} \otimes w_{1}^{A_{1}}\right) \oplus\left(w_{2}^{c} \otimes w_{2}^{A_{1}}\right) \\
& \oplus\left(w_{3}^{c} \otimes w_{3}^{A_{1}}\right) \oplus\left(w_{4}^{c} \otimes w_{4}^{A_{1}}\right) \\
& =\left(\left(\left(s_{5.8889}, 0.0190\right),\left(s_{0}, 0.1570\right)\right)\right. \\
& \left.\otimes\left(\left(s_{4.75},-0.0250\right),\left(s_{0.6667}, 0.0750\right)\right)\right)
\end{aligned}
$$

$$
\begin{aligned}
& \oplus\left(\left(\left(s_{5.5833},-0.0008\right),\left(s_{0.0556}, 0.1751\right)\right)\right. \\
& \left.\otimes\left(\left(s_{4.75},-0.0250\right),\left(s_{0.6667}, 0.0750\right)\right)\right) \\
& \oplus\left(\left(\left(s_{5.4444}, 0.2195\right),\left(s_{0.1667}, 0.2556\right)\right)\right. \\
& \left.\otimes\left(\left(s_{5.1667}, 0.1625\right),\left(s_{0.3333}, 0.1375\right)\right)\right) \\
& \oplus\left(\left(\left(s_{3.9167}, 0.3576\right),\left(s_{0.8889}, 0.2611\right)\right)\right. \\
& \left.\otimes\left(\left(s_{5.5833},-0.0250\right),\left(s_{0.3333},-0.0750\right)\right)\right) \\
& =\left(\left(s_{5.9698}, 0.1586\right),\left(s_{0}, 0.1945\right)\right)
\end{aligned}
$$

Similarly, we can determine the overall weights of the remaining alternatives as

$$
\begin{aligned}
& w_{2}^{A}=\left(\left(s_{5.9940}, 0.1242\right),\left(s_{0}, 0.1752\right)\right), \\
& w_{3}^{A}=\left(\left(s_{5.8911}, 0.2461\right),\left(s_{0}, 0.2226\right)\right) .
\end{aligned}
$$

In the following, the score values of the overall weights of alternatives are determined by using (11).

$$
\begin{aligned}
& S\left(w_{1}^{A}\right)=0.9945, \\
& S\left(w_{2}^{A}\right)=0.9952, \\
& S\left(w_{3}^{A}\right)=0.9929
\end{aligned}
$$

As $S\left(w_{2}^{A}\right)>S\left(w_{1}^{A}\right)>S\left(w_{3}^{A}\right)$, therefore, the ranking order of alternatives is $A_{2}>A_{1}>A_{3}$. This implies that $A_{2}$ is the most desirable alternative.

4.1. Comparative Analysis. In order to validate the feasibility of our proposed method, TOPSIS method is applied to solve the same problem. The results are shown as follows. 
TABLE 8: The aggregated matrix $\widehat{R}$.

\begin{tabular}{lcr}
\hline & $C_{1}$ & $C_{2}$ \\
\hline$A_{1}$ & $\left(\left(s_{4.75},-0.025\right),\left(s_{0.6667}, 0.075\right)\right)$ & $\left(\left(s_{4.75}, 0.45\right),\left(s_{0.3333}, 0.375\right)\right)$ \\
$A_{2}$ & $\left(\left(s_{5.5}, 0.225\right),\left(s_{0.1667}, 0.125\right)\right.$ & $\left(\left(s_{5.5}, 0.25\right),\left(s_{0.1667}, 0.125\right)\right)$ \\
$A_{3}$ & $\left(\left(s_{4.6667}, 0.15\right),\left(s_{0.75}, 0.15\right)\right.$ & $\left(\left(s_{4.333}, 0.299\right),\left(s_{0.75}, 0.4\right)\right)$ \\
\hline$A_{1}$ & $C_{3}$ & $C_{4}$ \\
$A_{2}$ & $\left(\left(s_{5.1667}, 0.1625\right),\left(s_{0.3333}, 0.1375\right)\right)$ & $\left(\left(s_{5.5833},-0.025\right),\left(s_{0.3333},-0.075\right)\right)$ \\
$A_{3}$ & $\left(\left(s_{5.5},-0.1375\right),\left(s_{0.833},-0.0875\right)\right)$ & $\left(\left(s_{5.5},-0.025\right),\left(s_{0.833}, 0.25\right)\right)$ \\
\hline
\end{tabular}

TABLE 9: The result of TOPSIS method.

\begin{tabular}{lcccc}
\hline Alternatives & $d_{i}^{+}$ & $d_{i}^{-}$ & $R C_{i}$ & Ranking result \\
\hline$A_{1}$ & 0.1175 & 0.8853 & 0.8828 & 2 \\
$A_{2}$ & 0.0594 & 0.9472 & 0.9410 & 1 \\
$A_{3}$ & 0.2269 & 0.7766 & 0.7739 & 3 \\
\hline
\end{tabular}

The elements of the aggregated matrix $\widehat{R}=\left(\widehat{r}_{i j}\right)_{3 \times 4}$ against criteria $C_{1}$ can be computed as in the following:

$$
\begin{aligned}
\widehat{r}_{11}= & \bigoplus_{q=1}^{3} b_{1 q}^{1}=b_{11}^{1} \oplus b_{12}^{1} \oplus b_{13}^{1} \\
= & \left(\left(s_{3}, 0.5\right),\left(s_{3}, 0.5\right)\right) \oplus\left(\left(s_{1}, 0.4\right),\left(s_{4}, 0.2\right)\right) \\
& \oplus\left(\left(s_{3},-0.5\right),\left(s_{2},-0.2\right)\right) \\
= & \left(\left(s_{4.75},-0.025\right),\left(s_{0.6667}, 0.075\right)\right) \\
\widehat{r}_{21}= & \bigoplus_{q=1}^{3} b_{2 q}^{1}=b_{21}^{1} \oplus b_{22}^{1} \oplus b_{23}^{1} \\
= & \left(\left(s_{4}, 0.2\right),\left(s_{1}, 0.4\right)\right) \oplus\left(\left(s_{3}, 0.5\right),\left(s_{3}, 0.5\right)\right) \\
& \oplus\left(\left(s_{3}, 0.1\right),\left(s_{2},-0.2\right)\right) \\
= & \left(\left(s_{5.5}, 0.225\right),\left(s_{0.1667}, 0.125\right)\right. \\
\widehat{r}_{31}= & \bigoplus_{q=1}^{3} b_{3 q}^{1}=b_{31}^{1} \oplus b_{32}^{1} \oplus b_{33}^{1} \\
= & \left(\left(s_{2},-0.2\right),\left(s_{3},-0.5\right)\right) \oplus\left(\left(s_{2},-0.2\right),\left(s_{3}, 0.1\right)\right) \\
& \oplus\left(\left(s_{3}, 0.5\right),\left(s_{3}, 0.5\right)\right) \\
= & \left(\left(s_{4.6667}, 0.15\right),\left(s_{0.75}, 0.15\right)\right.
\end{aligned}
$$

Similarly, by utilizing the I2TLPRs $B_{2}, B_{3}$, and $B_{4}$, we can get the remaining elements of $\widehat{R}$ against criteria $C_{2}, C_{3}$, and $C_{4}$, respectively. The final aggregated matrix $\widehat{R}=\left(\widehat{r}_{i j}\right)_{3 \times 4}$ can be seen as in Table 8 .

The intuitionistic 2-tuple positive ideal and negative ideal solutions are determined. The distances between each alternative to positive ideal alternative $d_{i}^{+}(i=1,2,3)$ and negative ideal alternative $d_{i}^{-}(i=1,2,3)$ are obtained by using (10). The weight values of the criteria are determined by calculating the score values of each element of $W_{C}=$ $\left(w_{1}^{c}, w_{2}^{c}, \ldots, w_{n}^{c}\right)$ with the help of (11). The relative weight denoted as $w_{j}(j=1,2,3,4)$ of each criterion is determined as follows:

$$
w_{i}=\frac{w_{j}^{c}}{\sum_{j=1}^{n} w_{j}^{c}}
$$

It can be observed that $\sum_{j=1}^{n} w_{j}=1$. The relative weights are determined as $w_{1}=0.2703, w_{2}=0.2612, w_{3}=0.2586$, and $w_{4}=0.2099$. The relative closeness coefficients $R C_{i}(i=$ $1,2,3)$ and ranking result can be seen in Table 9.

Again, the final ranking order is $A_{2}>A_{1}>A_{3}$ and the most desirable alternative is $A_{2}$.

It is apparent that results of I2TL AHP method and TOPSIS method are identical and the best and worst alternatives have no difference, which can illustrate the validity of our proposed method. As compared to the TOPSIS method, our method is more flexible. In addition, we can find that the proposed method considers bounded rationality of DMs in comparison with TOPSIS method. Obviously, the ranking result obtained by TOPSIS method may conform to the actual decision-making to some extent. As far as the time complexity is concerned, it is in general lower for AHP as compared with the TOPSIS method. The advantage of the AHP method over TOPSIS is that, in the AHP method, decision matrix consistency test is frequently needed. This leads to a consistent priority ranking with pairwise comparisons of the experts. Although both methods are equally adequate to deal with the lack of precision of scores of alternatives as well as the relative importance of different criteria, it is worth noting that the AHP method is more appropriate than the TOPSIS method when the purpose is to avoid the rank reversal phenomenon which lies at the heart of the main MCDM techniques like TOPSIS.

\section{Conclusion}

In this paper, intuitionistic 2-tuple AHP method has been proposed for solving the MCDM problems based on I2TLSs. 
Firstly, we have defined some operational laws for I2TLEs and proved some related important properties. Secondly, by using the idea of I2TLSs, two important preference relations, namely, the I2TLPR and the multiplicative I2TLPR, have been defined along with a transformation mechanism that can transform an I2TLPR to a corresponding IPR and vice versa. Thirdly, we have proposed an approach for checking the consistency of an I2TLPR and presented a method to repair the inconsistent one by using the proposed transformation mechanism. Finally, a comparative example is given to show the effectiveness of the proposed approach and is validated through a comparative analysis. The proposed approach is appropriate for a linguistic preference structure with symbolic translation parameters of linguistic arguments. Furthermore, the DMs remain much easier for collecting pairwise preference information using I2TLSs which are really effective in handling the vagueness and uncertainty in a MCDM problem. Our proposed intuitionistic 2-tuple AHP method is different from all the previous methods of decision-making because the proposed method uses I2TLSs, which always avoid any loss of information in the process. So it is an efficient and most feasible method for real-life applications of decision-making. On the basis of I2TLPRs, more applications should be worked on as our further research, for instance, performance evaluation, emergency management evaluation, and decision support systems, especially expert system.

\section{Data Availability}

The data used to support the findings of this study are included within the article.

\section{Conflicts of Interest}

The authors declare that they have no conflicts of interest.

\section{References}

[1] F. Herrera and L. Martínez, "An approach for combining linguistic and numerical information based on the 2-tuple fuzzy linguistic representation model in decision-making," International Journal of Uncertainty, Fuzziness and KnowledgeBased Systems, vol. 8, no. 5, pp. 539-562, 2000.

[2] F. Herrera and L. Martínez, "A 2-tuple fuzzy linguistic representation model for computing with words," IEEE Transactions on Fuzzy Systems, vol. 8, no. 6, pp. 746-752, 2000.

[3] F. Herrera, E. Herrera-Viedma, and L. Martínez, "A fusion approach for managing multi-granularity linguistic term sets in decision making," Fuzzy Sets and Systems, vol. 114, no. 1, pp. 43$58,2000$.

[4] F. Herrera and L. Martínez, "A model based on linguistic 2tuples for dealing with multigranular hierarchical linguistic contexts in multi-expert decision-making," IEEE Transactions on Systems, Man, and Cybernetics, Part B: Cybernetics, vol. 31, no. 2, pp. 227-234, 2001.

[5] J. Wang and J. Hao, "A new version of 2-tuple fuzzy linguistic representation model for computing with words," IEEE Transactions on Fuzzy Systems, vol. 14, no. 3, pp. 435-445, 2006.
[6] G. W. Wei, "Extension of TOPSIS method for 2-tuple linguistic multiple attribute group decision making with incomplete weight information," Knowledge and Information Systems, vol. 25, no. 3, pp. 623-634, 2010.

[7] I. Beg and T. Rashid, "Hesitant 2-tuple linguistic information in multiple attributes group decision-making," Journal of Intelligent and Fuzzy Systems, vol. 30, pp. 109-116, 2016.

[8] I. Beg and T. Rashid, "An Intuitionistic 2-Tuple Linguistic Information Model and Aggregation Operators," International Journal of Intelligent Systems, vol. 31, no. 6, pp. 569-592, 2016.

[9] P. Liu and S.-M. Chen, "Multiattribute group decision making based on intuitionistic 2-tuple linguistic information," Information Sciences, vol. 430/431, pp. 599-619, 2018.

[10] T. L. Saaty, The Analytic Hierarchy Process, McGraw-Hill, NY, USA, 1980

[11] L. F. D. O. M. Santos, L. Osiro, and R. H. P. Lima, "A model based on 2-tuple fuzzy linguistic representation and Analytic Hierarchy Process for supplier segmentation using qualitative and quantitative criteria," Expert Systems with Applications, vol. 79, pp. 53-64, 2017.

[12] W. Li, S. Yu, H. Pei, C. Zhao, and B. Tian, "A hybrid approach based on fuzzy AHP and 2-tuple fuzzy linguistic method for evaluation in-flight service quality," Journal of Air Transport Management, vol. 60, pp. 49-64, 2017.

[13] F. Tüysüz and B. Simsek, "A hesitant fuzzy linguistic term setsbased AHP approach for analyzing the performance evaluation factors: an application to cargo sector," in Complex \& Intelligent Systems DOI, vol. 3, pp. 167-175, 3 edition, 2017.

[14] F. Chiclana, F. Herrera, and E. Herrera-Viedma, "Integrating three representation models in fuzzy multipurpose decision making based on fuzzy preference relations," Fuzzy Sets and Systems, vol. 97, no. 1, pp. 33-48, 1998.

[15] F. Chiclana, E. Herrera-Viedma, F. Alonso, and S. Herrera, "Cardinal consistency of reciprocal preference relations: a characterization of multiplicative transitivity," IEEE Transactions on Fuzzy Systems, vol. 17, no. 1, pp. 14-23, 2009.

[16] M. Xia, Z. Xu, and H. Liao, "Preference relations based on intuitionistic multiplicative information," IEEE Transactions on Fuzzy Systems, vol. 21, no. 1, pp. 113-133, 2013.

[17] Z. Fan and X. Chen, "Consensus Measures and Adjusting Inconsistency of Linguistic Preference Relations in Group Decision Making," in Fuzzy Systems and Knowledge Discovery, vol. 3613 of Lecture Notes in Computer Science, pp. 130-139, Springer, Berlin, Germany, 2005.

[18] E. Herrera-Viedma, L. Martínez, F. Mata, and F. Chiclana, "A consensus support system model for group decision-making problems with multigranular linguistic preference relations," IEEE Transactions on Fuzzy Systems, vol. 13, no. 5, pp. 644-658, 2005.

[19] M. Delgado, J. L. Verdegay, and M. A. Vila, "On aggregation operations of linguistic labels," International Journal of Intelligent Systems, vol. 8, no. 3, pp. 351-370, 1993.

[20] Y. Dong, Y. Xu, and S. Yu, "Linguistic multiperson decision making based on the use of multiple preference relations," Fuzzy Sets and Systems, vol. 160, no. 5, pp. 603-623, 2009.

[21] F. Herrera, E. Herrera-Viedma, and J. L. Verdegay, "A sequential selection process in group decision making with a linguistic assessment approach," Information Sciences, vol. 85, no. 4, pp. 223-239, 1995.

[22] J. Kacprzyk, "Group decision making with a fuzzy linguistic majority," Fuzzy Sets and Systems, vol. 18, no. 2, pp. 105-118, 1986. 
[23] T. L. Saaty, "A scaling method for priorities in hierarchical structures," Journal of Mathematical Psychology, vol. 15, no. 3, pp. 234-281, 1977.

[24] V. Cutello and J. Montero, "Fuzzy rationality measures," Fuzzy Sets and Systems, vol. 62, no. 1, pp. 39-54, 1994.

[25] E. Herrera-Viedma, F. Herrera, F. Chiclana, and M. Luque, "Some issues on consistency of fuzzy preference relations," European Journal of Operational Research, vol. 154, no. 1, pp. 98$109,2004$.

[26] Z. Xu, X. Cai, and E. Szmidt, "Algorithms for estimating missing elements of incomplete intuitionistic preference relations," International Journal of Intelligent Systems, vol. 26, no. 9, pp. 787-813, 2011

[27] Y. Dong, Y. Xu, and H. Li, "On consistency measures of linguistic preference relations," European Journal of Operational Research, vol. 189, no. 2, pp. 430-444, 2008.

[28] Z. Xu and H. Liao, "Intuitionistic Fuzzy Analytic Hierarchy Process," IEEE Transactions on Fuzzy Systems, vol. 22, no. 4, pp. 1-14, 2015.

[29] B. Zhu and Z. Xu, "Consistency measures for hesitant fuzzy linguistic preference relations," IEEE Transactions on Fuzzy Systems, vol. 22, no. 1, pp. 35-45, 2014.

[30] Z. Zhang and C. Wu, "On the use of multiplicative consistency in hesitant fuzzy linguistic preference relations," KnowledgeBased Systems, vol. 72, pp. 13-27, 2014.

[31] Z.-W. Gong, L.-S. Li, J. Forrest, and Y. Zhao, "The optimal priority models of the intuitionistic fuzzy preference relation and their application in selecting industries with higher meteorological sensitivity," Expert Systems with Applications, vol. 38, no. 4, pp. 4394-4402, 2011.

[32] S.-P. Wan, Q.-Y. Wang, and J.-Y. Dong, "The extended VIKOR method for multi-attribute group decision making with triangular intuitionistic fuzzy numbers," Knowledge-Based Systems, vol. 52, pp. 65-77, 2013.

[33] N. Subramanian and R. Ramanathan, "A review of applications of Analytic Hierarchy Process in operations management," International Journal of Production Economics, vol. 138, no. 2, pp. 215-241, 2012.

[34] W. Ho, "Integrated analytic hierarchy process and its applications-a literature review," European Journal of Operational Research, vol. 186, no. 1, pp. 211-228, 2008.

[35] K. T. Atanassov, "Intuitionistic fuzzy sets," Fuzzy Sets and Systems, vol. 20, no. 1, pp. 87-96, 1986.

[36] K. Atanassov and G. Gargov, "Interval valued intuitionistic fuzzy sets," Fuzzy Sets and Systems, vol. 31, no. 3, pp. 343-349, 1989.

[37] Z. Xu, "Intuitionistic preference relations and their application in group decision making," Information Sciences, vol. 177, no. 11, pp. 2363-2379, 2007.

[38] F. Herrera, E. Herrera-Viedma, and J. L. Verdegay, "A model of consensus in group decision making under linguistic assessments," Fuzzy Sets and Systems, vol. 78, no. 1, pp. 73-87, 1996.

[39] F. Herrera and E. Herrera-Viedma, "Linguistic decision analysis: steps for solving decision problems under linguistic information," Fuzzy Sets and Systems, vol. 115, no. 1, pp. 67-82, 2000.

[40] Z. S. Xu, "A method based on linguistic aggregation operators for group decision making with linguistic preference relations," Information Sciences, vol. 166, no. 1-4, pp. 19-30, 2004.

[41] Z. S. Xu, "Group decision making based on multiple types of linguistic preference relations," Information Sciences, vol. 178, no. 2, pp. 452-467, 2008.
[42] X. Gou and Z. Xu, "Novel basic operational laws for linguistic terms, hesitant fuzzy linguistic term sets and probabilistic linguistic term sets," Information Sciences, vol. 372, pp. 407-427, 2016. 


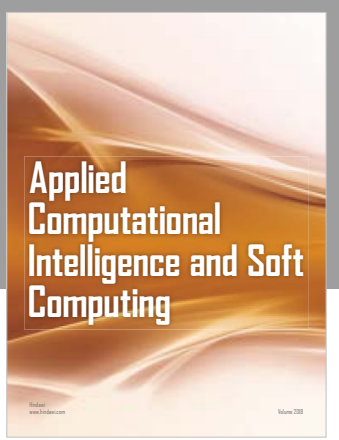

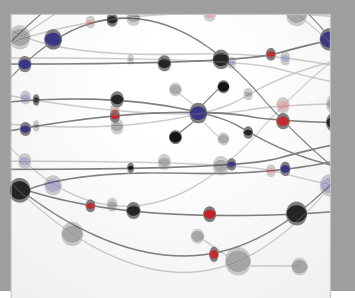

The Scientific World Journal
Submit your manuscripts at

Computing
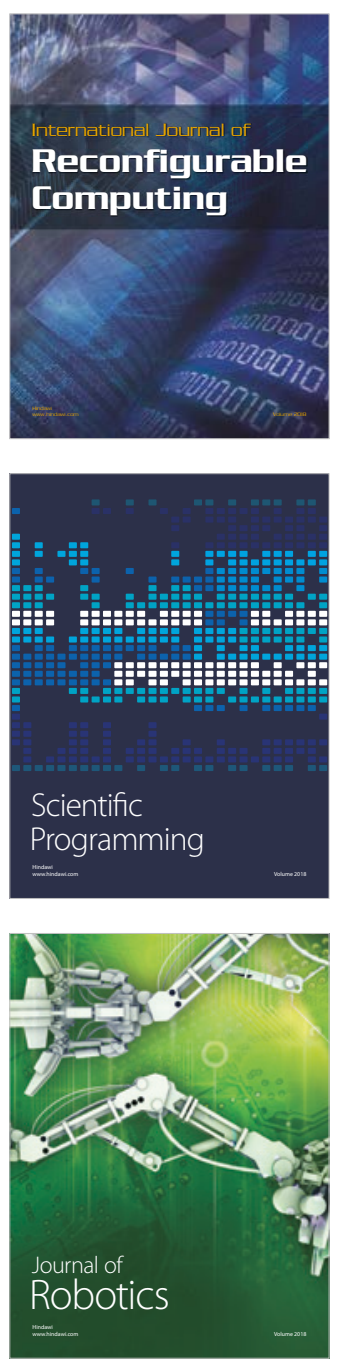

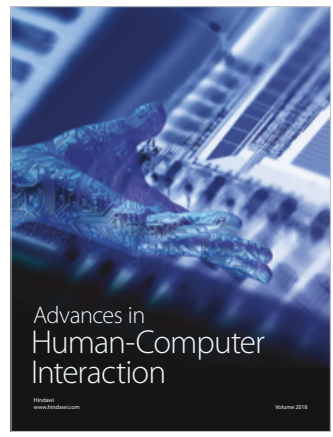

Human-Compute

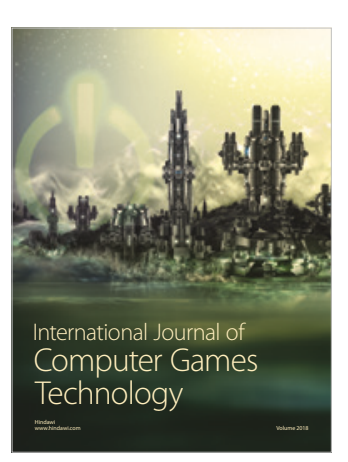

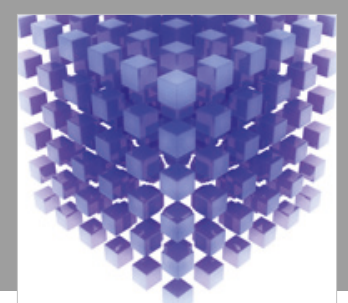

Mathematical Problems in Engineering

\section{Engincering}
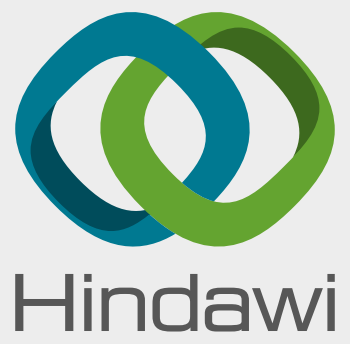

www.hindawi.com
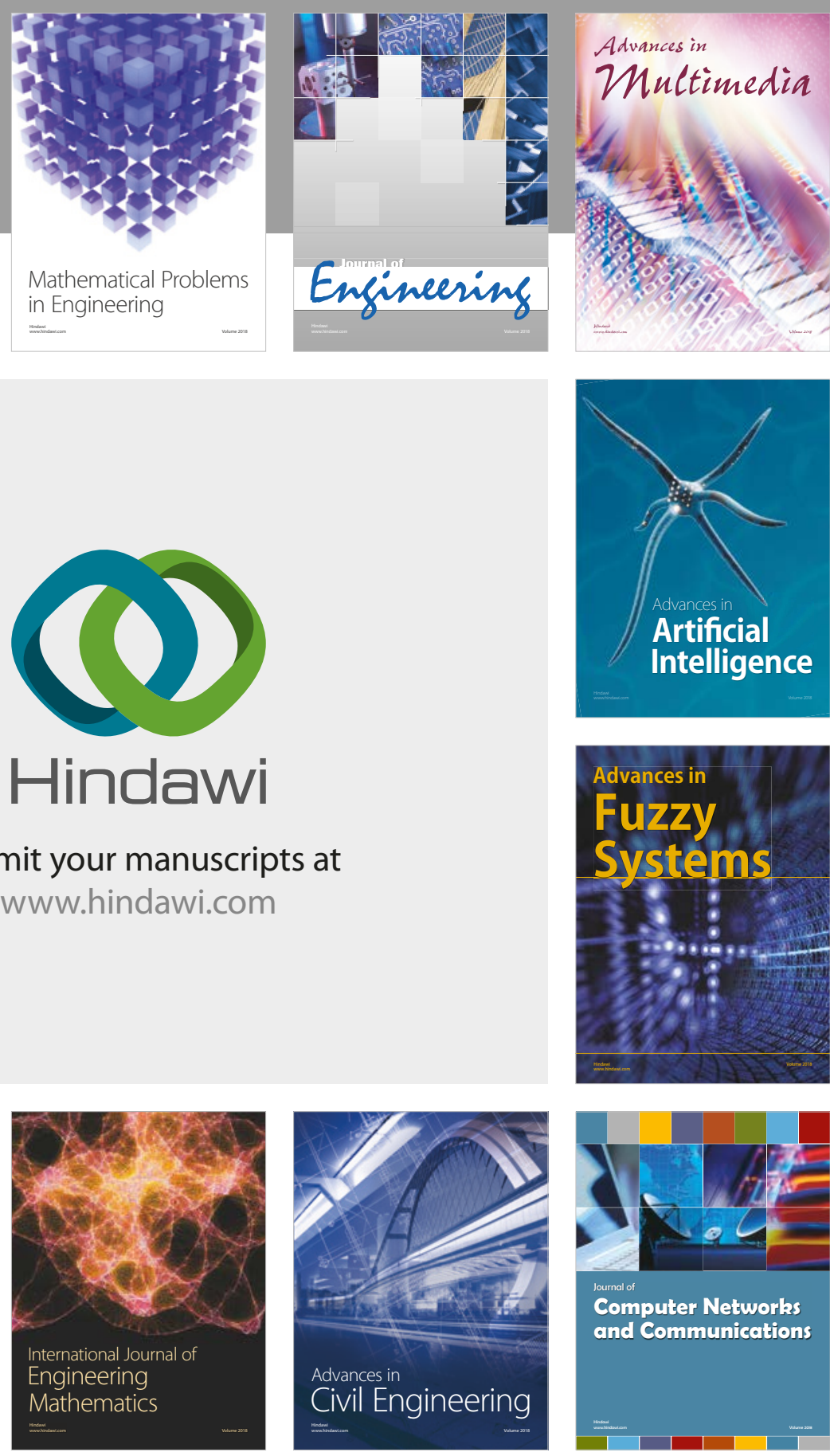

Computer Networks and Communications

Multimedia
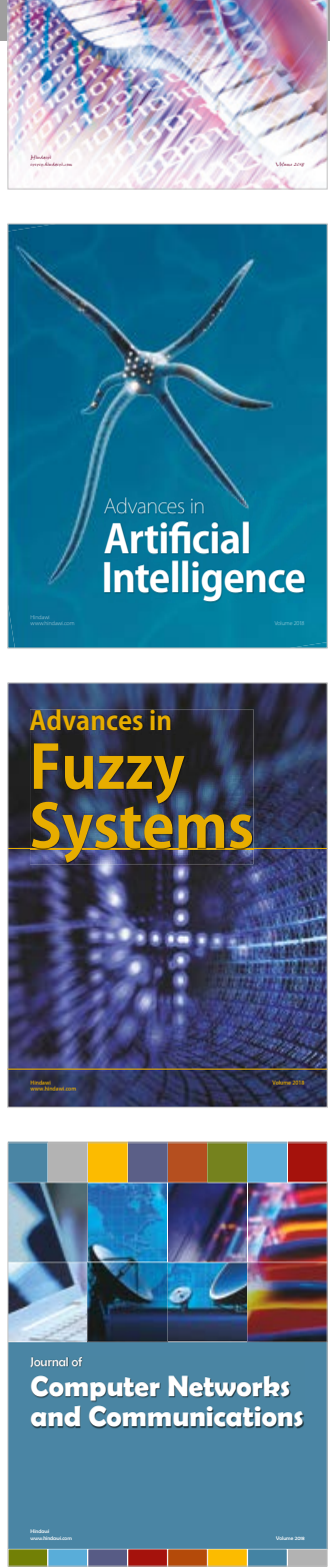

Advances in

Modelling \&

Simulation

in Engineering

interaction

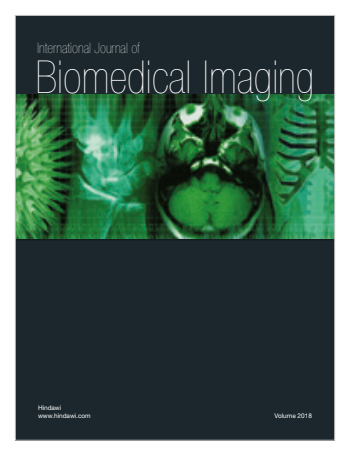

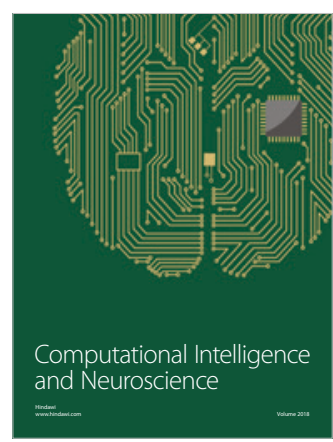

